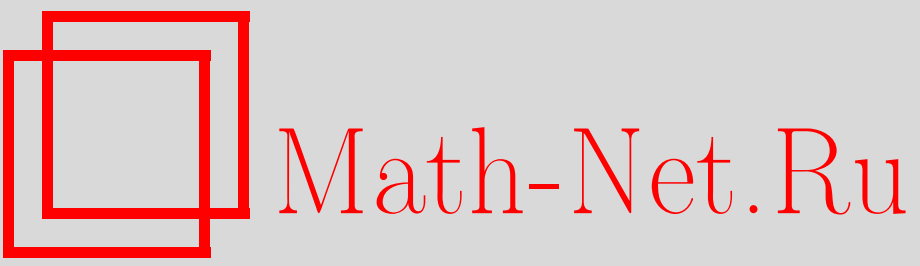

М. В. Куркина, В. В. Славский, Однородные функции на гильбертовом пространстве и квазиконформные преобразования сферы, Итоги науки и техн. Сер. Соврем. мат. и ее прил. Темат. обз., 2020, том 188, 70-75

DOI: https://doi.org/10.36535/0233-6723-2020-188-70-75

Использование Общероссийского математического портала Math-Net.Ru подразумевает, что вы прочитали и согласны с пользовательским соглашением

http: //www. mathnet.ru/rus/agreement

Параметры загрузки:

IP : 54.162 .85 .209

26 апреля 2023 г., 15:37:06 


\title{
ОДНОРОДНЫЕ ФУНКЦИИ НА ГИЛЬБЕРТОВОМ ПРОСТРАНСТВЕ И КВАЗИКОНФОРМНЫЕ ПРЕОБРАЗОВАНИЯ СФЕРЫ
}

\author{
(c) 2020 г. М. В. КУРКИНА, В. В. СЛАВСКИЙ \\ Посвящается академику Ю. Г. Решетняку
}

\begin{abstract}
АннотАция. При помощи однородных функций в гильбертовом пространстве в работе построен и исследован широкий класс квазиконформных преобразований сферы.

Ключевые слова: однородная функция, конформно-плоская метрика, квазиконформное отображение, преобразование Лежандра, конформно-выпуклая функция.
\end{abstract}

\section{HOMOGENEOUS FUNCTIONS ON HILBERT SPACES AND QUASICONFORMAL TRANSFORMATIONS OF A SPHERE}

\author{
(c) 2020 M. V. KURKINA, V. V. SLAVSKY
}

Abstract. By using homogeneous functions in a Hilbert space, a wide class of quasiconformal transformations of the sphere is constructed and examined.

Keywords and phrases: homogeneous function, conformally flat metric, quasiconformal mapping, Legendre transform, conformally convex function.

AMS Subject Classification: 53A07

1. Однородные функции и конформно-плоские метрики на сфере. Однородные функции играют важную роль в чистой математике (см. [2]) и её приложениях, например, в экономике при построении производственной функции (см. [1,3]). В [9] было замечено следующее свойство однородных функций.

Лемма 1. Пусть $f: \mathbb{R}^{n+1} \rightarrow \mathbb{R}$ - произвольная однородная степени 1 положительная функция на евклидовом пространстве $\mathbb{R}^{n+1}$, т.е. $f(\lambda x)=\lambda f(x)$ при $\lambda>0$, класса $C^{1}\left(\mathbb{R}^{n+1} \backslash\{0\}\right)$. Отображение $H_{f}: \mathbb{R}^{n+1} \rightarrow \mathbb{R}^{n+1}$, определяемое формулой

$$
H_{f}: \boldsymbol{x} \in \mathbb{R}^{n+1} \rightarrow \boldsymbol{x}-2 f(x) \frac{\nabla f}{|\nabla f|^{2}} \in \mathbb{R}^{n+1},
$$

сохраняет норму в $\mathbb{R}^{n+1}:\left\|H_{f}(x)\right\|=\|x\|$. Здесъ $\nabla f-$ градиент функции $f$ в $\mathbb{R}^{n+1}$.

Доказательство. В силу однородности функции $f$ выполняется равенство Эйлера $f(x)=(\boldsymbol{\nabla} f, \boldsymbol{x})$. Скалярный квадрат вектора $\boldsymbol{y}=H_{f}(\boldsymbol{x})$ равен

$$
(\boldsymbol{y}, \boldsymbol{y})=(\boldsymbol{x}, \boldsymbol{x})-4 f(x) \frac{(\boldsymbol{\nabla} f, \boldsymbol{x})}{|\nabla f|^{2}}+4 f^{2}(x) \frac{(\boldsymbol{\nabla} f, \boldsymbol{\nabla} f)}{|\nabla f|^{4}}=(\boldsymbol{x}, \boldsymbol{x}) .
$$

Работа выполнена при поддержке Российского фонда фундаментальных исследований (проекты №o 18-47860016, 18-01-00620) и Научного Фонда Югорского государственного университета (проект № 13-01-20/10). 
С однородной функцией $f$ на $\mathbb{R}^{n+1}$ степени 1 естественно связана конформно-плоская метрика на единичной сфере $S^{n} \subset \mathbb{R}^{n+1}$ :

$$
d s^{2}=\frac{d x^{2}}{f^{2}(x)}, \quad x \in S^{n}
$$

и полярная к ней метрика (см. [9])

$$
d s^{* 2}=\frac{d y^{2}}{f^{* 2}(y)}, \quad y \in S^{n}
$$

определяемая явной формулой

$$
f^{*}(y)=\max _{x \in S^{n}} \frac{\|x-y\|^{2}}{2 f(x)},
$$

где $\|x-y\|^{2}$ - квадрат хордового расстояния между точками $x, y \in S^{n}$. Формула (4) напоминает классическое определение преобразования Лежандра выпуклой функции $h(x)$ в евклидовом пространстве:

$$
h^{*}(y)=\max _{x \in \mathbb{R}^{n}}[(x, y)-h(x)]
$$

если вычитание заменить на деление. Поэтому имеет смысл формулу (4) называть преобразованием Лежандра конформно-плоской метрики (см. [6-8]).

Кроме явных формул (4), функции $f(x)$ и $f^{*}(y)$ могут выражаться друг через друга посредством формул параметрического вида:

$$
\begin{aligned}
& f^{*}(y)=\frac{2 f(x)}{|\nabla f(x)|^{2}}, \quad \boldsymbol{y}=\boldsymbol{x}-2 f(x) \frac{\nabla f(x)}{|\nabla f(x)|^{2}}=H_{f}(x), \\
& f(x)=\frac{2 f^{*}(y)}{\left|\nabla f^{*}(y)\right|^{2}}, \quad \boldsymbol{x}=\boldsymbol{y}-2 f^{*}(y) \frac{\nabla f^{*}(y)}{\left|\nabla f^{*}(y)\right|^{2}}=H_{f^{*}}(y) .
\end{aligned}
$$

Определение 1. Оператор $H_{f}$ назовём конформным градиентом функции $f$ (см. [9]). Отображение $H_{f}$ инволютивно, т.е. $H_{f}^{-1}=H_{f}$.

Пример 1. Пусть $f(x)=a|x|+(b, x)$, где $a>0$ - число, $b \in \mathbb{R}^{n+1}$ - вектор. Тогда

$$
\begin{gathered}
\nabla f(x)=a \frac{x}{|x|}+b, \quad|\nabla f|^{2}(x)=a^{2}+2 \frac{a(x, b)}{|x|}+(b, b), \\
H_{f}(x)=\frac{x\left((b, b)-a^{2}\right)-2(a|x|+(b, x)) b}{a^{2}+2 a \frac{(x, b)}{|x|}+(b, b)} .
\end{gathered}
$$

Замечание 1. Формулу (1) можно использовать в более общей ситуации

$$
H_{V}(Z)=Z-2(Z, V) \frac{V}{|V|^{2}} \in \mathbb{R}^{n+1},
$$

где $V$-произвольный ненулевой (не фиксированный) вектор; здесь также для всех $Z \in \mathbb{R}^{n+1}$ выполняется равенство $\left\|H_{V}(Z)\right\| \equiv\|Z\|$. Преобразование $H_{V}(Z)$ есть просто симметрия $Z$ относительно гиперплоскости, перпендикулярной вектору $V$; если использовать терминологию из [10], то $H_{V}$ - «зеркало» в однородном пространстве $S^{n}$.

Замечание 2. Формулы (1)-(4) верны также в случае произвольной функции $f(x), x \in \overline{\mathbb{R}^{n}}$, без требования её однородности.

\section{2. Коэффициент квазиконформности отображение $H_{f}$.}

Определение 2. Пусть $f: S^{n} \rightarrow S^{n}$-диффеоморфизм единичной сферы, $x \in S^{n}, y=f(x)$, $d f_{x}: T_{x} \rightarrow T_{y}$ - дифференциал $f$ в точке $x$. Дилатацией $f$ в точке $x \in S^{n}$ называется число

$$
q(x, f)=\frac{\max _{|\xi|=1}\left\|d f_{x}(\xi)\right\|}{\min _{|\xi|=1}\left\|d f_{x}(\xi)\right\|}=\frac{\lambda_{\max }}{\lambda_{\min }},
$$


где $\lambda_{\max }, \lambda_{\min }$ - максимальное и минимальное собственные значения линейного оператора $\sqrt{d f_{x}^{*} \circ d f_{x}}$. Коэфбициентом квазиконформности $f$ называется число $q(f)=\max _{x} q(x, f)$.

Лемма 2. Пусть $d s^{2}=d x^{2} / f^{2}(x)$ - конформно-плоская метрика, определенная на сфере $S^{n}$, $x \in S^{n}, y=H_{f}(x), d H_{f_{x}}: T_{x} \rightarrow T_{y}-$ дифференииал $H_{f}$ в точке $x$. Тогда на единичных касательных векторах $\xi$ выполняется равенство

$$
\left|d H_{f}(\xi)\right|=\frac{2\left|K_{1 / 2}(f, x, \xi)\right|}{|\nabla f|^{2}},
$$

где

$$
K_{1 / 2}(f, x, \xi)=f \frac{d^{2} f}{d \xi^{2}}-\frac{1}{2}|\nabla f|^{2}
$$

- одномерная секционная кривизна конформно-плоской метрики (см. [7,8]). Здесь $d^{2} f / d \xi^{2}-в т о-$ рая производная функиии в точке $x \in \mathbb{R}^{n}$ вдоль единичного вектора $\xi, \nabla f$-градиент функции $f$ в $\mathbb{R}^{n}$. Формула верна как в плоском случае, так и для единичной сферы, в этом случае функиия $f: S^{n} \rightarrow \mathbb{R}$ продолжсается по однородности на $\mathbb{R}^{n+1}, x \in S^{n} \subset \mathbb{R}^{n+1}, \xi$-единичный вектор, касательный к сфере в точке $x, \nabla f$-градиент функиии в $\mathbb{R}^{n+1}$.

Доказательство. Дифференцируя равенство

$$
H_{f}(\boldsymbol{x})=\boldsymbol{x}-2 f(x) \frac{\nabla f}{|\nabla f|^{2}}
$$

вдоль касательного вектора $\xi \in T_{x}$, получим

$$
d H_{f}(\xi)=\xi-2(\nabla f, \xi) \frac{\nabla f}{|\nabla f|^{2}}-2 f\left[\frac{d(\nabla f)(\xi)}{|\nabla f|^{2}}-\frac{2 \nabla f(\nabla f, d(\nabla f)(\xi))}{|\nabla f|^{4}}\right] .
$$

Введем следующий линейный оператор $P: T_{x} \rightarrow T_{x}$ на касательном пространстве $T_{x}$ :

$$
P(v)=v-2 \frac{\nabla f}{|\nabla f|}\left(v, \frac{\nabla f}{|\nabla f|}\right) .
$$

Оператор $P$ есть симметрия относительно подпространства, перпендикулярного вектору $\nabla f$. Тогда

$$
\begin{aligned}
d H_{f}(\xi)=P(\xi)-\frac{2 f}{|\nabla f|^{2}} P(d(\nabla f)(\xi))=P\left(\xi-\frac{2 f d(\nabla f)(\xi)}{|\nabla f|^{2}}\right) & = \\
& =-\frac{2}{|\nabla f|^{2}} P\left[f d(\nabla f)(\xi)-\frac{1}{2}|\nabla f|^{2} \xi\right] .
\end{aligned}
$$

Заметим, что

$$
K_{1 / 2}(f, x, \xi)=\left(\xi, f d(\nabla f)(\xi)-\frac{1}{2}|\nabla f|^{2} \xi\right) .
$$

Так как $P$ - изометричный оператор на единичных касательных векторах $\xi$, то выполняется равенство

$$
\left|d H_{f}(\xi)\right|=\frac{2\left|K_{1 / 2}(f, x, \xi)\right|}{|\nabla f|^{2}},
$$

что и требовалось.

Из формулы (7) непосредственно вытекает следующее утверждение.

Теорема 1. Если конформно-плоская метрика $d s^{2}=d x^{2} / f^{2}(x)$ имеет положительную одномерную кривизну, то дилатация $H_{f}$ в точке $x \in S^{n}$ равна

$$
q\left(x, H_{f}\right)=\frac{\max _{i}\left\{k_{i}(x)\right\}}{\min _{j}\left\{k_{j}(x)\right\}},
$$




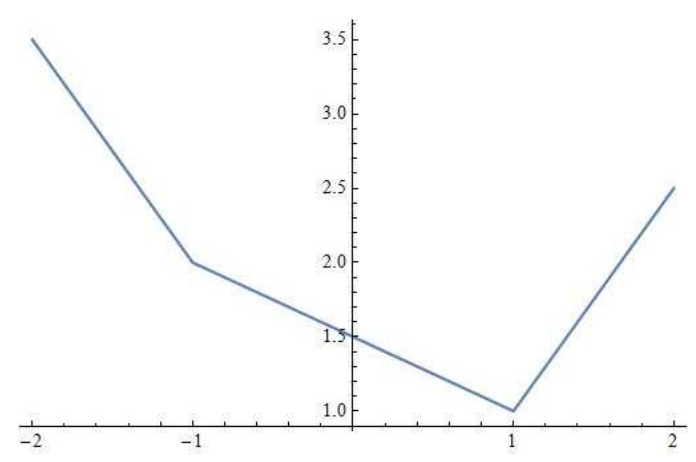

Рис. 1. График функции $\delta(x)$

где $\left\{k_{1}(x), \ldots, k_{n}(x)\right\}$ - главные значения одномерной секиионной кривизны $K_{1 / 2}(f, x, \xi)$. Коэ фичиент квазиконформности отображения $H_{f}$ равен

$$
q\left(H_{f}\right)=\max _{x \in S^{n}} \frac{\max _{i}\left\{k_{i}(x)\right\}}{\min _{j}\left\{k_{j}(x)\right\}} .
$$

Замечание 3. Неотрицательность одномерной секционной кривизны конформно-плоской метрики эквивалентна выполнению трёхточечному неравенству - условию конформной выпуклости функции $g(x)=\sqrt{f(x)}$ на сфере $($ см. $[7,8]$ :

$$
g(x) \leqslant g\left(x_{1}\right) \frac{\left|x_{2}-x\right|}{\left|x_{2}-x_{1}\right|}+g\left(x_{2}\right) \frac{\left|x-x_{1}\right|}{\left|x_{2}-x_{1}\right|}
$$

это позволяет конструктивно строить квазиконформные отображения сферы.

Пример 2. Пусть $x_{1}, x_{2} \in \mathbb{R}^{n}, a, b>0$. Функция

$$
\delta(x)=\frac{a\left|x_{2}-x\right|}{\left|x_{2}-x_{1}\right|}+\frac{b\left|x_{1}-x\right|}{\left|x_{2}-x_{1}\right|}
$$

является конформно-выпуклой (это следует из неравенства Птолемея). Функцию такого вида назовем диполем с вершинами в точках $x_{1}, x_{2}$. На рис. 1 показан график функции $\delta(x)$ для размерности 1.

Одномерная секционная кривизна метрики $d s^{2}=d x^{2} / \delta(x)^{4}$ диполя равна

$$
K_{1 / 2}(x, \xi)=\frac{2 \delta^{2}(x) a b}{\left|x_{2}-x_{1}\right|^{2}\left|x_{2}-x\right|\left|x_{1}-x\right|}\left[\left|x_{2}-x_{1}\right|^{2}-\left(\frac{x_{1}-x}{\left|x_{1}-x\right|}\left|x_{2}-x\right|-\frac{x_{2}-x}{\left|x_{2}-x\right|}\left|x_{1}-x\right|, \xi\right)^{2}\right] .
$$

Так как

$$
\left|\frac{x_{1}-x}{\left|x_{1}-x\right|}\right| x_{2}-x\left|-\frac{x_{2}-x}{\left|x_{2}-x\right|}\right| x_{1}-x||^{2}=\left|x_{2}-x_{1}\right|^{2},
$$

то согласно неравенству Коши-Буняковского получим

$$
\left|\left(\frac{x_{1}-x}{\left|x_{1}-x\right|}\left|x_{2}-x\right|-\frac{x_{2}-x}{\left|x_{2}-x\right|}\left|x_{1}-x\right|, \xi\right)\right| \leqslant\left|x_{2}-x_{1}\right||\xi| .
$$

Следовательно, для одномерной кривизны диполя получим оценки

$$
0 \leqslant K_{1 / 2}(x, \xi) \leqslant \frac{2 \sqrt{a b}}{\left|x_{2}-x_{1}\right|^{2}}\left(\sqrt{\frac{a\left|x_{2}-x\right|}{\left|x_{1}-x\right|}}+\sqrt{\frac{b\left|x_{1}-x\right|}{\left|x_{2}-x\right|}}\right)^{2} .
$$

Одномерная секционная кривизна обращается в нуль в направлении

$$
\xi \uparrow \frac{x_{1}-x}{\left|x_{1}-x\right|}\left|x_{2}-x\right|-\frac{x_{2}-x}{\left|x_{2}-x\right|}\left|x_{1}-x\right|
$$

и принимает максимальное значение в перпендикулярном направлении. 


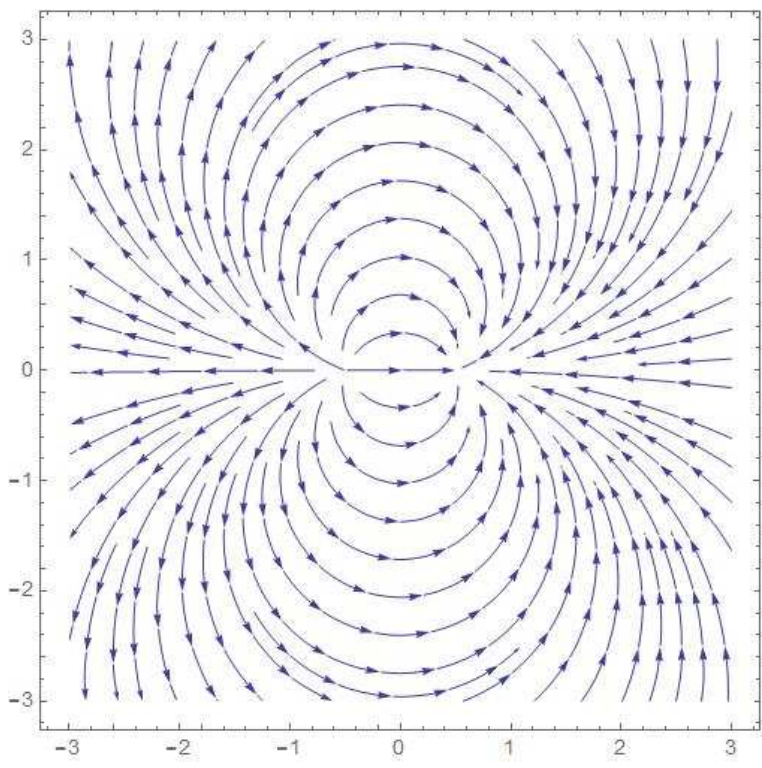

Рис. 2. Поле направлений с нулевой кривизной метрики $d s^{2}=d x^{2} / \delta^{4}(x)$

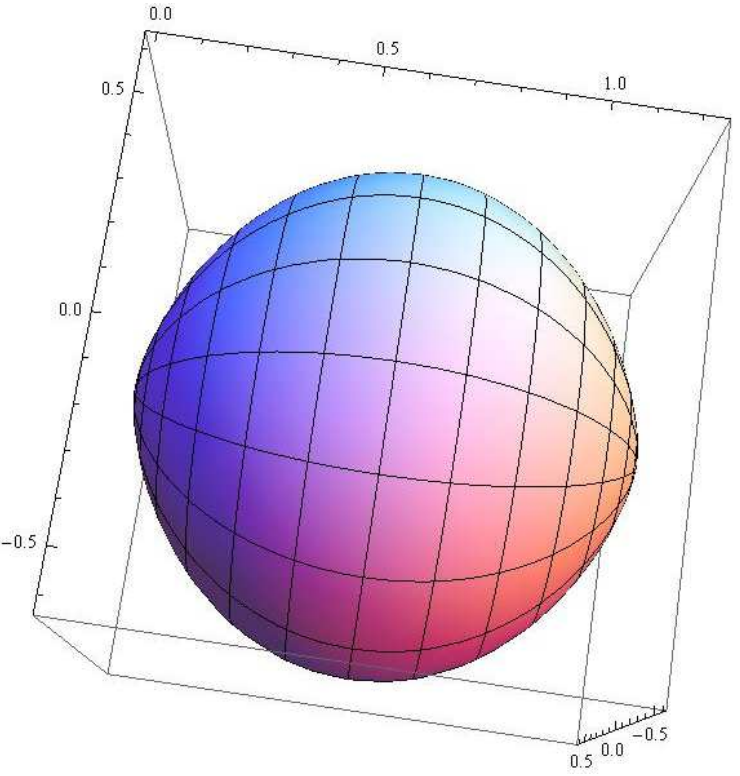

Рис. 3. Поверхность в $\mathbb{R}^{3}$, отвечающая метрике $d s^{2}=d x^{2} / \delta^{4}(x)$

Следствие 1. В силу теоремы 1 коэфбициент квазиконформности отображения $y=H_{\delta}(x)$ обращается в бесконечность в точках, где одномерная секиионная кривизна имеет направления с нулевым значением, поэтому для метрики диполя это верно во всех точек, т.е. точки конформности отсутствуют. На рис. 2 показано поле направлений с нулевой одномерной кривизной метрики $d s^{2}=d x^{2} / \delta^{4}(x)$.

Следствие 2. Для размерности 2 гауссова кривизна дипольной метрики в точке $x \in \overline{\mathbb{R}^{2}}$ равна сумме одномерных кривизн в двух перпендикулярных направлениях и равна

$$
G=\frac{2 \sqrt{a b}}{\left|x_{2}-x_{1}\right|^{2}}\left(\sqrt{\frac{a\left|x_{2}-x\right|}{\left|x_{1}-x\right|}}+\sqrt{\frac{b\left|x_{1}-x\right|}{\left|x_{2}-x\right|}}\right)^{2} .
$$

Следовательно, выполняется неравенство

$$
G \geqslant \frac{2 \sqrt{a b}}{\left|x_{2}-x_{1}\right|^{2}} 4 \sqrt{a b}=\frac{8 a b}{\left|x_{2}-x_{1}\right|^{2}},
$$

и двумерная метрика $d s^{2}=d x^{2} / \delta^{4}(x)$ имеет положстельную гауссову кривизну.

Замечание 4. Для размерности 2 из работ Ю. Г. Решетняка (см. [4]) следует интегральное представление метрики положительной гауссовой кривизны в виде логарифмического потенциала

$$
f(x)=\exp \left[\int \log |x-y| d \mu_{y}\right] .
$$

Нетрудно проверить, что потенциал Рисса

$$
f(x)=\int|x-y| d \lambda_{y}
$$

определяет конформно-плоскую метрику неотрицательной одномерной кривизны (конформновыпуклую функцию). Возникает естественная задача доказать это представление для произвольной конформно-выпуклой функции.

Теорема А. Д. Александрова о реализации метрики положительной кривизны выпуклой поверхностью в соединении с теоремой А. В. Погорелова о регулярности выпуклой поверхности 
с регулярной метрикой дали полное решение проблемы Вейля о реализации в трехмерном евклидовом пространстве регулярной метрики положительной кривизны. Можно непосредственно проверить, что метрика $d s^{2}=d x^{2} / \delta^{4}(x)$ принадлежит классу $C^{2}$, за исключением двух точек $x_{1}$, $x_{2}$, в которых она непрерывна, и ей соответствует поверхность вращения циклоиды (рис. 3), с двумя особыми точками, в которых поверхность принадлежит классу $C^{1+0.5}$, имеет касательную плоскость, а гауссова кривизна стремится к $+\infty$.

\section{СПИСОК ЛИТЕРАТУРЫ}

1. Вольных Е. В., Кутышкин А. В., Никоноров Ю. Г. Построение $\delta$-однородной производственной VESфункции // Сиб. ж. индустр. мат. - 2007. - 10, № 2 (30). - С. 31-44.

2. Гельфанд И. М., Шапиро З. Я. Однородные функции и их приложения// Усп. мат. наук. $-1955 .-$ 10, № 3. - С. 3-70.

3. Кутышкин A. В., Сокол Г. А. О применении производственных функций вида VES-функция для моделирования функционирования экономических систем// Вестн. ЮУрГУ. Сер. Компьютерные технологии. Управление. Радиоэлектроника. - 2007. - 17, № 1. - С. 49-60.

4. Решетняк Ю. Г. Двумерные многообразия ограниченной кривизны// Итоги науки и техн. Сер. Совр. пробл. мат. Фундам. направл. - 1989. - 70. - С. 7-189.

5. Славский В. В. Оценка коэффициента квазиконформности области через кривизну квазигиперболической метрики// Сиб. мат. ж. - 1999. - 40, № 4. - С. 947-965.

6. Kurkina M. V. The generalized polar transform conformally-flat metrics of positive one-dimensional curvature// Proc. Int. Conf. «Geometric Analysis» (September 22-28, 2019, Novosibirsk). - Novosibirsk: Novosibirsk State Univ., 2019. - C. 89-91.

7. Kurkina M. V., Rodionov E. D., Slavsky V. V. Conformally convex functions and conformally flat metrics of nonnegative curvature// Dokl. Math. - 2015. - 91, № 3. - C. 287-289.

8. Kurkina M. V., Rodionov E. D., Slavsky V. V. Legendre transformation of conformal convex functions// Proc. Int. Conf. «Geometric Analysis» (September 22-28, 2019, Novosibirsk). - Novosibirsk: Novosibirsk State Univ., 2019. - C. 118-120.

9. Rodionov E. D., Slavsky V. V. Polar transform of conformally flat metrics// Sib. Adv. Math. - 2018. 28, № 2. - C. 101-114.

10. Sabinin L. V. Mirror Geometry of Lie Algebras, Lie Groups, and Homogeneous Spaces. - Springer, 2004.

Куркина Мария Викторовна

Югорский государственный университет, Ханты-Мансийск

E-mail: mavi@inbox.ru

Славский Виктор Владимирович

Югорский государственный университет, Ханты-Мансийск

E-mail: slavsky2004@mail.ru 